\title{
Novel Bidirectional Multiple-Input Multiple-Output Converter for Simultaneous Direct Battery Module Balancing
}

\author{
Reinhold Koch ${ }^{1 *}$, Andreas Jossen ${ }^{2}$, Robert Kuhn ${ }^{1}$ \\ *reinhold.koch@tum.de \\ ${ }^{I}$ TUM CREATE, 1 CREATE Way, Singapore 138602, Singapore \\ ${ }^{2}$ Institute for Electrical Energy Storage Technology, Technische Universität München, Karlstr. 45, 80333 Munich, \\ Germany
}

\begin{abstract}
Charge balancers are needed to equalize the energy stored in battery cells connected in series to increase the usable capacity and lifetime of the entire stack. A multiple-input multiple-output DC/DC converter for battery module balancing, based on flux additivity, is proposed. It allows bidirectional, simultaneous and direct charge transfer. A prototype converter with two bidirectional channels has successfully been built-up and tested. First experimental results are presented to verify the feasibility of the charge balancer. A time averaged model is derived considering the influence of the common multiwinding transformer voltage.
\end{abstract}

Keywords: charge equalization, Battery Management System, DC-DC, converter, electronic

\section{Introduction}

Energy storage in electrochemical storage systems has received increasing interest in the last years, as traction batteries for the propulsion of electric vehicles or as stationary batteries for compensating the fluctuating supplies of renewable energy sources. These two applications require high energy content and power capability from the battery. Therefore the battery cells are connected in series in order to provide a favourable voltage for the motor or the grid. Due to fabrication tolerances and different operating conditions of the cells in the battery pack, the characteristics of each single cell vary from one to the other. One important characteristic is the capacity of the cell. In a stack of cells the capacity of the weakest cell determines the overall usable capacity. This is aggravated by the fact that this cell experiences higher depth of discharge and, during operation, will age and loose its capacity quicker than the others [1].

\section{Regenerative Cell Balancing Concepts}

One solution to this problem is cell balancing - the charge and discharge of single cells in order to equilibrate the energy content of all cells. The simplest approach is dissipative active balancing by connecting each cell to a resistor with a switch. This only allows a limited balancing speed and imbalance, since the resistors only can be placed on a printed circuit board when they dissipate a few $100 \mathrm{~mW}$. At higher power the resistors have to be actively cooled. In order to use the stored energy to its full extent and to achieve higher energy efficiencies regenerative charge balancing has to be used which also allows balancing during discharging. All types of regenerative balancing methods use an interim storage device for transferring the energy from one cell to the other, 
like a capacitor, inductor or the inductance of a transformer. In several review papers these balancing methods are often grouped according to this interim storage device [2]. But from an application point of view they should be rather grouped according to the characteristics of the energy flow and influence on the charge balancing strategy. The most important of these characteristics are whether the energy transfer can be done bidirectional and simultaneous as well as the energy transfer path. For the energy transfer path there are basically three different types of concepts: stepwise, stack and direct charge balancing.

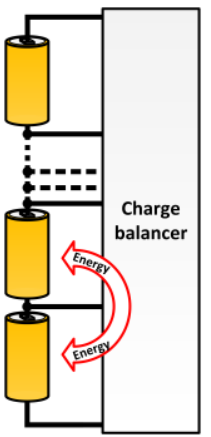

(a)

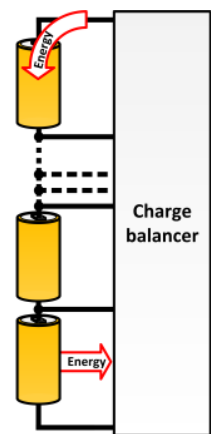

(b)

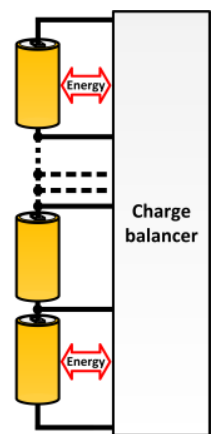

(c)
Figure 1: Charge balancing concepts (a) stepwise, (b) stack and (c) direct balancing:

\subsection{Stepwise Balancing}

The first concept only allows an energy exchange between adjacent cells. For nonadjacent cells the energy has to be transferred stepwise from one cell over several others to the target cell. Since every step is penalized with losses, the balancing efficiency can be heavily affected. The benefit of this method is that the different input ports of the balancing structure do not have to be isolated against each other. Also the necessary blocking voltage of the switches can be kept low. Typical representatives of this kind of charge balancers are the Buck-Boost converter [3], the Ćuk converter [4] as well as the switched capacitor [5].

\subsection{Stack Balancing}

The next approach uses the fact that all cells are connected in series in the stack. The charge balancer converts the discharged energy from an input port to a common output port which is then feeding into the whole stack. The energy flow can be also the other way around. The drawback of this method is the inherently limited balancing efficiency since the energy which just has been discharged from one cell will be partly charged back again into the same cell when feeding the discharged energy into the stack. Representatives are the Flyback converter [6] or more elaborated transformer based solutions [7].

\subsection{Direct Balancing}

The last method is the most convenient, but technically also the most complex solution. A direct charge balancing allows discharging any cell and feeding this energy into any other cell. This is only possible if all the ports are isolated or the switches have a sufficient blocking voltage to switch the interim storage device to all the voltage levels in the stack. One representative of this kind of charge balancer is the flying capacitor or also called the switched capacitor with a single capacitor [8]. Other approaches to the concept of direct balancing require an enormous amount of switches, setup in a matrix structure, which at this extent becomes unreasonable.

\subsection{Bidirectional Operation}

The energy flow for stepwise balancing should be bidirectional since it is not known in advance if charge balancing in the stack has to be done from top to bottom or vice versa.

Also for direct balancing the ports have to be bidirectional since it is not known in advance which will be the cell with the highest and which will be the one with the lowest capacity.

The benefit of stack balancing is that the input ports as well as the output ports can be unidirectional. The choice of direction, from the single cell into the stack or from the stack to the single cell, is making either balancing during charging or discharging more effective.

\subsection{Simultaneous Operation}

Stepwise balancing concepts usually operate simultaneously, but most of the stack and direct balancer concepts do not allow simultaneous operation. In these cases the energy flow has to be multiplexed giving every port a certain time slot to exchange its energy. For a charge balancer with $n$ ports the current during that time has then to be $\mathrm{n}$ times higher to compensate for the limited time. This will require to oversize the components. Additionally the losses inside the batteries and along any ohmic resistances increase quadratically with the withdrawn current. Therefore simultaneous operation of charge balancing should be preferred especially for applications like fast 
charging for which also the balancing speed is critical.

\subsection{Requirements for the proposed balancing system}

For the above stated reasons, the best concept is a system which allows bidirectional direct charge balancing simultaneously. We describe the topology of the converter we propose for such a system in section 3. Section 4 shows the operation principle. In section 5 we derive a time averaged model of the converter with various channels.

Due to its complexity, we only propose such a system for charge balancing between modules, consisting usually of 12 cells in series. Due to its simplicity and the possibility of bidirectional and simultaneous charge transfer, most of the stepwise balancing methods could be an option for cell balancing inside a battery module. The fabrication tolerances and the temperature gradients among the cells in a module are easier to keep low and therefore the overall necessity for balancing can be kept rather small, so that the reduced efficiency for balancing nonadjacent cells is acceptable.

\section{Design Specifications}

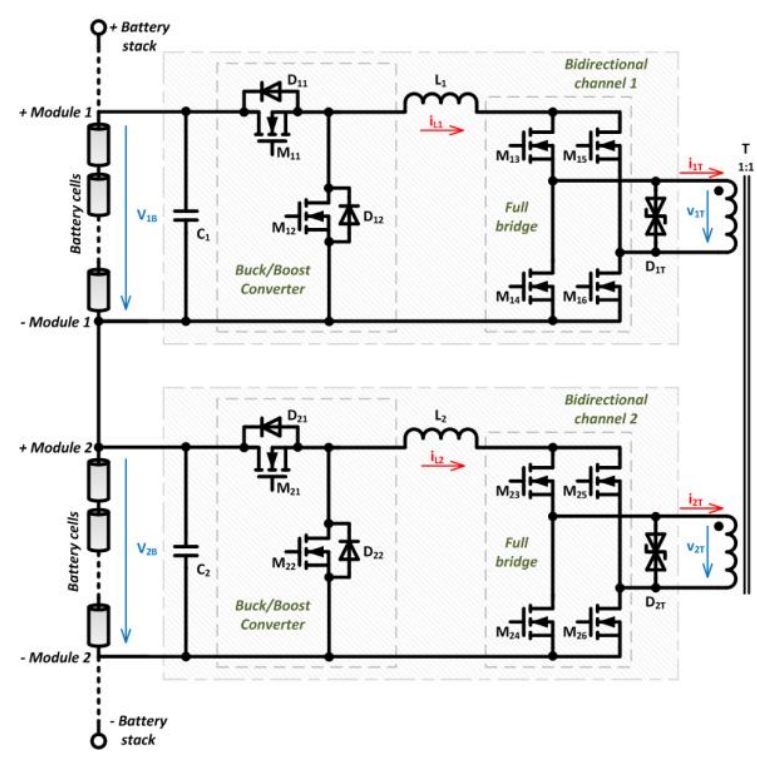

Figure 2: Topology of the proposed charge balancer

Figure 2 shows the topology of our prototype with two channels. The two bidirectional channels are connected to battery modules which are connected in series since the main battery current flows over this series connection and not over the MIMO converter itself. $\mathrm{M}_{\mathrm{x} 1}$ and $\mathrm{D}_{\mathrm{x} 2}$ of the bidirectional channel create a buck converter allowing the transfer of energy from the battery module to the transformer, while $\mathrm{M}_{\mathrm{x} 2}$ and $\mathrm{D}_{\mathrm{x} 1}$ create a boost converter from the transformer to the battery module allowing energy transfer in the opposite direction. The capacitor after $\mathrm{L}_{\mathrm{x}}$, which usually is used in buck/boost converter topologies, is omitted, making this first stage a natural current source. In order to alternate the voltage for power conversion over the transformer a full bridge is added $\left(M_{x 3}\right.$ to $\left.M_{x 6}\right)$. The transformer consists of 2 windings, with an equal turn ratio. The coils of the transformer overlap each other in order to create good coupling and low stray inductances. The suppressor diodes $\mathrm{D}_{\mathrm{xT}}$ at the terminals of each transformer winding limit the voltage induced after each half switching cycle of the full bridge (see section 4.3).

The MIMO converter was built up with each channel designed to be connected to a module with 12 lithium ion cells in series thus with up to a voltage of $50 \mathrm{~V}$. It is considered for a fast charging application with $360 \mathrm{~A}$ charging current. It has a current rating of $20 \mathrm{~A}$ and is therefore able to balance a maximum imbalance of $5 \%$ from the mean capacity of all modules. The following components and switching frequencies were used:

1) $M_{x 1}-M_{x 6}:$ Semikron SK85MH10T

2) Switching frequency $f_{s}=20 \mathrm{kHz}$

3) $\mathrm{L}_{\mathrm{x}}=160 \mu \mathrm{H}$

4) Transformer

a) core: $\mathrm{TX} 107 / 65 / 18-3 \mathrm{~F} 4$

b) turn ratio $=20: 20$

c) main Inductance $\mathrm{L}_{\mathrm{H}}=400 \mu \mathrm{H}$

d) stray Inductance $\mathrm{L}_{\sigma} \approx 1 \mu \mathrm{H}$

Since all channels are built the same way, it is easy to extend the converter to the desired amount of input/output ports.

\section{Operation Principle}

\subsection{Basic Operation}

During operation the full bridge FETs $M_{x 3}$ to $M_{x 6}$ are synchronized and operated simultaneously with a fixed duty cycle of ideally $50 \%$. Since the full bridge is fed by a current source, it has to be avoided that both switches in one leg (e.g. $\mathrm{M}_{\mathrm{x} 3}$ and $\mathrm{M}_{\mathrm{x} 4}$ ) are turned off at the same time. Due to the turn-on and turn-off time of the switches the duty cycles have to overlap a certain dead time during which both switches are conducting. This short circuits the current source and the transformer for a short period of time. The buck/boost converters of both bidirectional channels $\left(\mathrm{M}_{\mathrm{x} 1}, \mathrm{D}_{\mathrm{x} 2}\right.$ and $\mathrm{M}_{\mathrm{x} 2}$, 

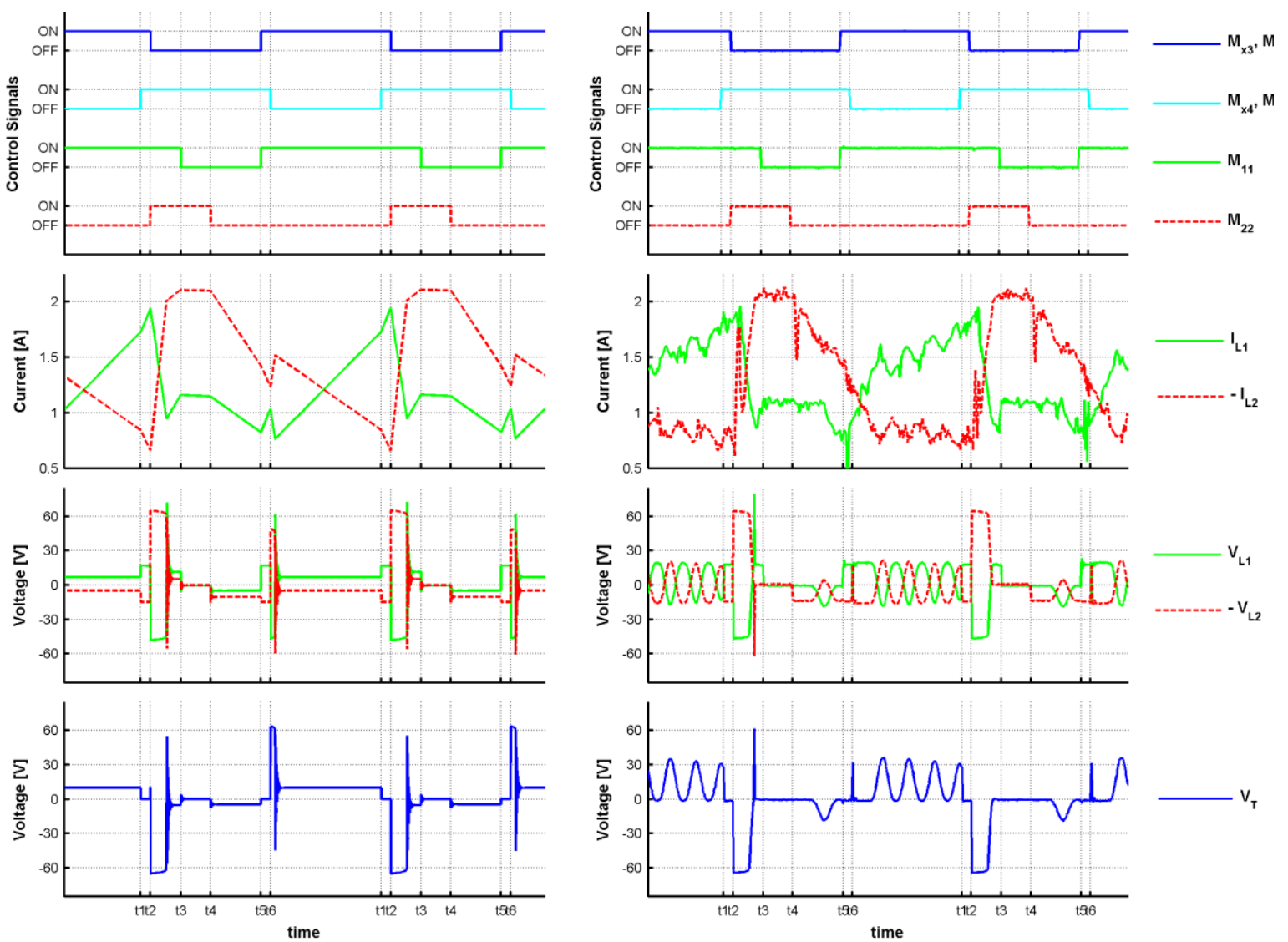

(a)

(b)

Figure 3: Results of an idealised simulation (a) and measurements (b) of the control signals (top; $\mathbf{M}_{\mathrm{x} 3}, \mathbf{M}_{\mathrm{x} 6}, \mathbf{M}_{\mathrm{x} 4}$, $\mathrm{M}_{\mathrm{x} 5}, \mathrm{M}_{\mathrm{x} 1}, \mathrm{M}_{\mathrm{x} 2}$ ) the inductor currents (second from top; $\mathrm{I}_{\mathrm{L} 1}-\mathrm{I}_{\mathrm{L} 2}$ ), the inductor voltages (third from top, $\mathrm{V}_{\mathrm{L} 1},-\mathrm{V}_{\mathrm{L} 2}$ ) for the discharging channel 1 (green solid) and charging channel 2 (red dashed) as well as the transformer voltage (bottom, $\mathrm{V}_{\mathrm{T}}$ ).

$\mathrm{D}_{\mathrm{x} 1}$ ) define the direction and the magnitude of the energy flow.

The current of the two bidirectional converter channels are combined by the fluxes in the coupled transformer core. Like this every channel can feed energy into or draw energy from the common transformer, as long as the current balance is considered.

From the discharged battery module, the energy is first converted to a current source and then fed on the common multiwinding transformer, which creates a voltage source again. The other channel converts the voltage of the transformer to a current source in order to charge the connected battery module. The change between voltage and current source is a commonly used concept. Although in this case the conversion from a current source to a voltage source is not done by a capacitor but with a transformer. In this way no time constant is added to the system which simplifies the system dynamics and control.

\subsection{Operating stages}

Different operating stages are shown in Figure 3 with an energy flow from channel 1 to channel 2 by only operating the buck converter MOSFET $\mathrm{M}_{11}$ of channel 1 and the boost converter MOSFET $\mathrm{M}_{22}$ of channel 2. MOSFET $\mathrm{M}_{12}$ and $\mathrm{M}_{21}$ are turned off for the whole sequence, since the current direction is not changed. Please note that the inductor current and voltage of channel 2 are negative and shown with the direction from the transformer to the battery module. For the reasons of clarity the waveforms on the left side are taken from a simulation with ideal values and behaviour. The right side shows the measurement data of the real converter. The waveforms are explained section by section:

5) (t1 $\leq \mathrm{t}<\mathrm{t} 2)$ : The transformer is short circuited and $\mathrm{M}_{11}$ is on, therefore the input voltage is increasing the current in the inductor of channel 1. In channel 2 the same occurs in the 
opposite direction. In this direction the current can flow over $\mathrm{D}_{21}$, feeds into the battery module and is decreasing.

6) (t2 $\leq \mathrm{t}<\mathrm{t} 3$ ): After each half switching cycle of the bridge switches the current in the leakage inductance $\mathrm{L}_{\sigma}$ of the transformer has the opposite direction as the current in the main inductor $\mathrm{L}_{\mathrm{x}}$. This induces a voltage which is limited to around 66 Volts by the suppressor diodes $\mathrm{D}_{\mathrm{xT}}$. The negative voltage difference over the Inductor $\mathrm{L}_{1}$ is decreasing its current. The positive voltage difference over $\mathrm{L}_{2}$ additionally with the turned on boost FET $\mathrm{M}_{22}$ increases the current in the inductor $\mathrm{L}_{2}$.

7) $(\mathrm{t} 3 \leq \mathrm{t}<\mathrm{t} 4): \mathrm{M}_{11}$ is turned off and with it the driving force for any current in the system. At the same time the current in the second channel is freewheeling over $\mathrm{M}_{22}$ and the bridge MOSFETs $\mathrm{M}_{24}$ and $\mathrm{M}_{25}$. For these reasons the current does not change in channel 1 nor does it in channel 2.

8) $(\mathrm{t} 4 \leq \mathrm{t}<\mathrm{t5})$ : The boost MOSFET $\mathrm{M}_{22}$ is turned off, allowing the energy stored in the inductor $\mathrm{L}_{2}$ to charge the battery module 2 . This decreases the current through $\mathrm{L}_{2}$ and $\mathrm{L}_{1}$.

9) (t5 $\leq \mathrm{t}<\mathrm{t} 6)$ : The transformer is again shorted and $M_{11}$ is turned on. These are the same conditions as under 1 )

10) $(\mathrm{t} 6 \leq \mathrm{t}<\mathrm{t} 1)$ : This time the voltage spike is smaller. Decreasing and increasing the current $\mathrm{I}_{\mathrm{L} 1}$ and $\mathrm{I}_{\mathrm{L} 2}$ respectively only for a short while before the in average positive voltage over $\mathrm{L}_{1}$ increases $\mathrm{I}_{\mathrm{L} 1}$ and the in average negative voltage over $\mathrm{L}_{2}$ decreases $\mathrm{I}_{\mathrm{L} 2}$.

\subsection{Discussion of the induced voltage after full bridge switching}

The reasons for the induced voltage after each half switching cycle of the full bridge are offsets in the current balance and the current in the leakage inductance of the transformer, whose direction has to be changed each half switching cycle. The associated energy which is stored in the leakage inductance is dissipated in the suppressor diodes $D_{\mathrm{xT}}$. This reduces the efficiency of the converter. Several measures can help reducing this otherwise lost energy:

- Reducing the leakage inductance $L_{\sigma}$ itself by providing good coupling between the transformer windings.

- Keep the magnetizing current of the transformer low by increasing its main inductance $\mathrm{L}_{\mathrm{H}}$. The magnetizing current has to be provided by the feeding channels and therefore leads to a difference of the current balance of the common multiwinding transformer.

- Avoid increases of the current in the main inductor $\mathrm{L}_{\mathrm{x}}$ of each channel during the short circuit of the full bridge by turning the buck MOSFETs $\mathrm{M}_{\mathrm{x} 1}$ of the feeding channels off and the boost MOSFETs $\mathrm{M}_{\mathrm{x} 2}$ of the sinking channels on as shown in Figure 4 Like this the current in the main inductor $\mathrm{L}_{\mathrm{x}}$ is freewheeling during the time of the short circuit.

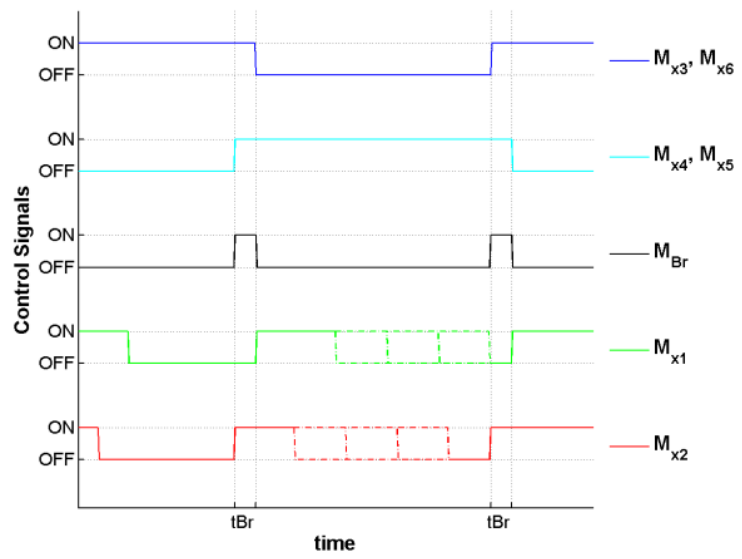

Figure 4: Switching pattern for reduced voltage clamping

- The voltage could be also clamped to the battery module at the input or to any other voltage level in the stack partially recuperating the energy in this way.

\section{Time Averaged Model}

The most important value to be controlled is the current of each channel. It can be controlled by changing the voltage over the main inductor $\mathrm{L}_{\mathrm{x}}$. As seen in section 4 , this value changes depending on the switches and the transformer voltage, making its exact description very complex. As proposed in [9], instead of describing the actual current waveform, it is sufficient to determine a description averaged over one switching cycle. Since a digital control is sampling the current also only once in a switching cycle this can be called a sampled-data model and is sufficient for a later control design. In this way, especially the description of the transformer voltage $\mathrm{V}_{\mathrm{T}}$ becomes very simple since the whole topology of the MIMO converter can be reduced to the circuit in Figure 5.

If all main inductances $L_{x}$ have the same value, the averaged transformer voltage can be calculated by

$$
V_{T}=\frac{\sum_{x=1}^{n} V_{x B} *}{n}
$$




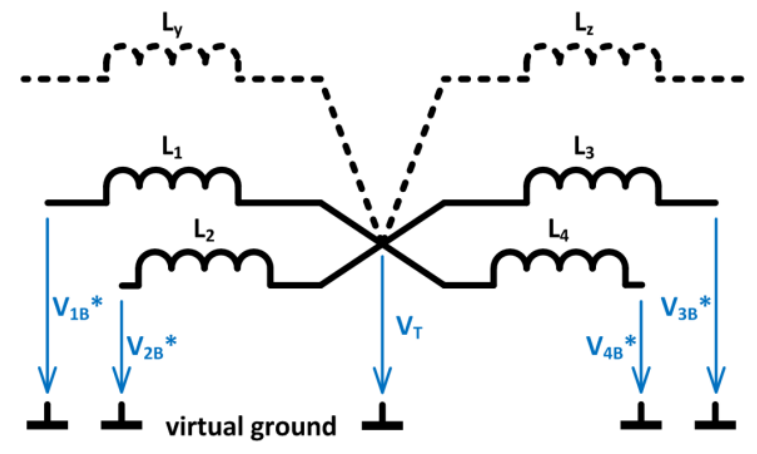

Figure 5: Simplified schematic for the averaged modell of the transformer voltage

where $\mathrm{n}$ is the number of converter channels and $V_{x B} *$ is the effective battery module voltage calculated by

$$
V_{x B} *=V_{x B} \cdot d_{x 1} \cdot\left(1-d_{x 2}\right) \cdot\left(1-d_{B r}\right)
$$

where $d_{x 1}$ and $d_{x 2}$ are respectively the duty cycles of $\mathrm{M}_{\mathrm{x} 1}$ and $\mathrm{M}_{\mathrm{x} 2}$ considering that a $100 \%$ would not include the time when all switches of the full bridge are turned on. $d_{B r}$ is the short circuit duty cycle of the full bridge. This is true no matter at what time these switches are turned on, because they only determine when the battery voltage is connected to the main inductor $\mathrm{L}_{\mathrm{x}}$ on the left side, whose voltage can be assumed to be of constant value. The transformer voltage is always present on the right side of the inductor $\mathrm{L}_{\mathrm{x}}$, since it cannot be switched on or off, and therefore can be simplified by its average. Equation (2) assumes that $\mathrm{M}_{\mathrm{x} 1}$ is only turned on for positive currents and $\mathrm{M}_{\mathrm{x} 2}$ for negative currents. With this averaged model the schematic of each channel can be simplified to the one shown in Figure 6 and the change of the inductor current becomes

$$
\frac{d I_{L x}}{d t}=\frac{\left(V_{x B}-V_{T}\right)}{L_{x}}
$$

\section{Conclusion and Outlook}

A bidirectional MIMO Converter for direct simultaneous battery module balancing has been proposed. Basic power-stage circuit operation has

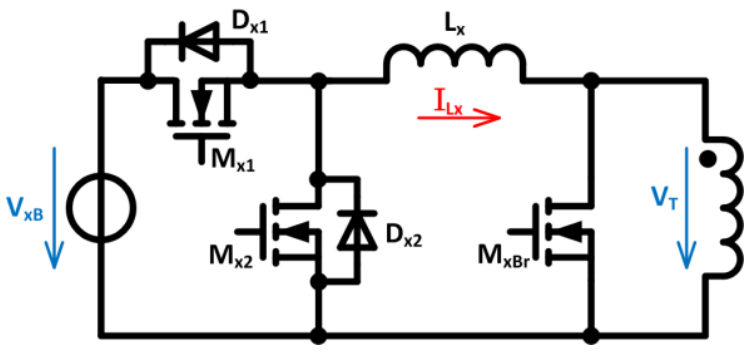

Figure 6: Simplified schematic for the averaged modell of the converter been described and an averaged model has been developed. Future work will include a control method based on the model derived and a comparison of the efficiency with other charge balancing methods.

\section{Acknowledgements}

This work was financially supported by the Singapore National Research Foundation under its Campus for Research Excellence And Technological Enterprise (CREATE) programme.

\section{References}

[1] G. Sarre, "Aging of lithium-ion batteries", Journal of Power Sources, Volume 127, Issues 1-2, 10 March 2004, Pages 65-71, ISSN 0378-7753

[2] M. Daowd, "Passive and active battery balancing comparison based on MATLAB simulation," Vehicle Power and Propulsion Conference (VPPC), 2011 IEEE, pp.1-7, 6-9 Sept. 2011

[3] N.H. Kutkut, "A modular nondissipative current diverter for EV battery charge equalization," APEC '98. Conference Proceedings 1998., Thirteenth Annual, vol.2, pp.686-690 vol.2, 15-19 Feb 1998

[4] Y.-S. Lee, "Battery Equalization Using Bidirectional Cuk Converter in DCVM Operation," 2005. PESC '05. IEEE 36th, pp.765-771, 16 June 2005

[5] C. Pascual, "Switched capacitor system for automatic series battery equalization," APEC '97 Conference Proceedings 1997, Twelfth Annual, vol.2, pp.848-854, 23-27 Feb 1997

[6] N.H. Kutkut, "Nondissipative current diverter using a centralized multi-winding transformer," PESC '97 Record, 28th Annual IEEE, vol.1, pp.648-654 vol.1, 22-27 Jun 1997

[7] Y.-M. Chen, "Multi-input DC/DC converter based on the multiwinding transformer for renewable energy applications," IEEE Transactions on Industry Applications, vol.38, no.4, pp.1096-1104, Jul/Aug 2002

[8] M.J. Isaacson, "Advanced lithium ion battery charger," Battery Conference on Applications and Advances, 2000. The Fifteenth Annual, pp.193-198, 11-14 Jan. 2000

[9] D. Maksimovic et al., "Modeling and simulation of power electronic converters," Proceedings of the IEEE, vol.89, no.6, pp.898-912, Jun 2001 


\section{Authors}

Reinhold Koch received his M. Sc. From the Technical University of Munich in 2011. At the moment he is a PhD student at TUM CREATE in Singapore. His research interests are Power Electronics in battery management systems, online diagnosis and fast charging.

Prof. Dr.-Ing. Andreas Jossen earned his doctorate, dealing with "management of photovoltaic plants using energy storage systems" at University of Stuttgart. Since 1994 he was group leader for different battery related topics at ZSW in Ulm. Since 2010 he is full professor at the Institute for Electrical Energy Storage Technology at the Technical University of Munich.

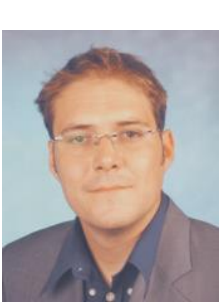

Dr. Robert Kuhn received his doctorate degree from the University in Ulm on the water management of fuel cells. He worked as a group leader for battery pack engineering at the ZSW in Ulm. At the moment he is leading the battery pack group of TUM CREATE in Singapore. 